\title{
Soluble Adenylyl Cyclase Activity Is Necessary for Retinal Ganglion Cell Survival and Axon Growth
}

\author{
Raul G. Corredor, ${ }^{1,2}$ Ephraim F. Trakhtenberg, ${ }^{1,2}$ Wolfgang Pita-Thomas, ${ }^{1}$ Xiaolu Jin, ${ }^{1}$ Ying Hu, ${ }^{1}$ \\ and Jeffrey L. Goldberg ${ }^{1,2}$ \\ ${ }^{1}$ Bascom Palmer Eye Institute, Interdisciplinary Stem Cell Institute, and ${ }^{2}$ Neuroscience Program, University of Miami Miller School of Medicine, Miami, \\ Florida 33136
}

cAMP is a critical second messenger mediating activity-dependent neuronal survival and neurite growth. We investigated the expression and function of the soluble adenylyl cyclase (sAC, ADCY10) in CNS retinal ganglion cells (RGCs). We found sAC protein expressed in multiple RGC compartments including the nucleus, cytoplasm and axons. sAC activation increased cAMP above the level seen with transmembrane adenylate cyclase (tmAC) activation. Electrical activity and bicarbonate, both physiologic sAC activators, significantly increased survival and axon growth, whereas pharmacologic or siRNA-mediated sAC inhibition dramatically decreased RGC survival and axon growth in vitro, and survival in vivo. Conversely, RGC survival and axon growth were unaltered in RGCs from AC1/AC8 double knock-out mice or after specifically inhibiting tmACs. These data identify a novel sAC-mediated cAMP signaling pathway regulating RGC survival and axon growth, and suggest new neuroprotective or regenerative strategies based on sAC modulation.

\section{Introduction}

Why do CNS neurons fail to regenerate and ultimately die after injury? Understanding the signal transduction pathways controlling neuronal survival and axon growth may lead to therapies for CNS injury and neurodegenerative diseases. One important and ubiquitous second messenger, cAMP is critical to both survival and axon growth as well as numerous processes including cellular differentiation, proliferation, and migration, among others (Grandoch et al., 2010). In developing and adult neurons, cAMP also mediates effects due to electrical activity and calcium influx (Spitzer, 2006; Root et al., 2008). For example, electrical activity promotes retinal ganglion cell (RGC) survival and axon growth by a cAMP-dependent mechanism (Shen et al., 1999; Goldberg et al., 2002) that enhances RGC responsiveness to growth factors (Meyer-Franke et al., 1998; Shen et al., 1999; Miotke et al., 2007; Zhao et al., 2009). Such data raise the hypothesis that calcium-activated adenylate cyclases (ACs) AC1, $\mathrm{AC}$, and the recently described soluble AC (sAC, also named AC10)

\footnotetext{
Received 0ct. 19, 2011; revised April 8, 2012; accepted April 11, 2012.

Author contributions: R.G.C., E.F.T., W.P.-T., Y.H., and J.L.G. designed research; R.G.C., E.F.T., W.P.-T., X.J., and Y.H. performed research; R.G.C., E.F.T., W.P.-T., X.J., Y.H., and J.L.G. analyzed data; R.G.C., E.F.T., W.P.-T., X.J., Y.H., and J.L.G. wrote the paper.

We gratefully acknowledge funding from the National Eye Institute (Grants EY022129 and P30-EY014801), NINDS (Grants NS061348 and T32-NS007492), American Heart Association (0535364N and 11Pre7610007), James and Esther King Foundation, and an unrestricted grant from Research to Prevent Blindness to the University of Miami. J.L.G. is the Walter G. Ross Distinguished Chair in Ophthalmic Research, and R.G.C. and E.F.T. are Lois Pope LIFE Fellows. We are indebted for the generous gifts of reagents from Marla Feller (University of California, Berkeley, CA) and Dan Storm (University of Washington, Seattle, WA; AC1/8 knock-out mice), Weidong Geng (University of Texas, Dallas. TX; anti-human SAC antibody), and Lexicon Genetics, Inc. (Woodlands, TX; brain and eye paraffin sections from Lex/Sacytm 1 knock-out and WT mice). We thank Michael Steketee for helpful comments on the manuscript, and Dale Brown, Shaoyun Zhang, and Scott Jordan for technical assistance.

The authors declare no competing financial interests.

Correspondence should be addressed to Jeffrey L. Goldberg, University of Miami Miller School of Medicine, 1501 NW $10^{\text {th }}$ Ave, BRB 826, Miami, FL 33136. E-mail: jgoldberg@med.miami.edu.

DOI:10.1523/JNEUROSCI.5288-11.2012

Copyright $\odot 2012$ the authors $\quad 0270-6474 / 12 / 327734-11 \$ 15.00 / 0$
}

may mediate these activities. Transmembrane ACs (tmACs) including AC1 and AC8 have been well studied and CAMP was thought to be generated solely by tmACs in RGCs, but recent findings suggest that cAMP elevation in response to activity may also originate from other as-yet unidentified sources (Dunn et al., 2009).

sAC, originally described in testis, is found from cyanobacteria to mammals, including plants, and is activated by basic chemical compounds including calcium, bicarbonate, ATP, and $\mathrm{CO}_{2}$ (Lomovatskaya et al., 2008). A putative sAC knock-out (KO) mouse showed only infertility and dyslipidemia (Farrell et al., 2008). Subsequent work demonstrated that this mouse does express other sAC isoforms in somatic cells (Farrell et al., 2008), and many groups have since documented sAC functions in the cytoplasm, mitochondria, and nuclei of several mammalian cell types (Zippin et al., 2004, 2010; Han et al., 2005; Schmid et al., 2007; Acin-Perez et al., 2009b; Geng et al., 2009; Hallows et al., 2009; Kumar et al., 2009; Strazzabosco et al., 2009; Chen et al., 2010; Păunescu et al., 2010; Tresguerres et al., 2010; Li et al., 2011). sAC may modulate netrin1-mediated axon guidance in dorsal root ganglion cells (Wu et al., 2006), and sAC is detectable in mammalian CNS neurons, but with unknown function (Farrell et al., 2008). Therefore, sAC may modulate cAMP-mediated functions previously attributed to tmACs. Here we show that sAC, but not tmACs 1 and 8, is necessary for RGC survival and axon growth in vitro and survival in vivo.

\section{Materials and Methods}

Detailed Materials and Methods are available upon request. Experiments conformed to the ARVO Statement for the Use of Animals in Ophthalmic and Vision Research and were approved by the Institutional Animal Care and Use Committee and the Institutional Biosafety Committee at the University of Miami.

Cell culture and reagents. Reagents were purchased from Sigma (Sigma- Aldrich) unless specified. RGCs were purified from early post- 
natal (P1-P9) Sprague Dawley rats, and WT or AC1/AC8 double KO mice, in collaboration with M. Feller (University of California, Berkeley, CA) (Dunn et al., 2009) and D. Storm (University of Washington, Seattle, WA) (Wong et al., 1999), by immunopanning, as described previously (Meyer-Franke et al., 1995), using antibodies against Thyl (T11D7 from ATCC for rat, F7D5 from AbD Serotec for mouse). Unless otherwise stated, RGCs were cultured in growth medium as described previously (Meyer-Franke et al., 1995), in Neurobasal medium (Invitrogen) supplemented with sodium pyruvate, $N$-acetyl cysteine, B-27, L-glutamine, Sato supplement, insulin, BDNF, and CNTF, with or without forskolin. For experiments testing sAC activity, the medium used during RGC purification was substituted with bicarbonate-free DMEM incubated overnight on $10 \% \mathrm{CO}_{2}$, after which the incubated bicarbonate-free DMEM (Invitrogen catalog \#31600-034), supplemented with D-glucose ( $4.5 \mathrm{~g} / \mathrm{L}$ ), and HEPES ( $25 \mathrm{~mm}$ ), was used to prepare bicarbonate concentrations from 0 to $40 \mathrm{~mm}$. Each condition was adjusted to physiologic $\mathrm{pH} 7.2$ with $\mathrm{NaOH}$ for bicarbonate-free medium and $\mathrm{HCl}$ for the bicarbonatecontaining medium, allowing medium $\mathrm{pH}$ to equilibrate in the incubator for additional $30 \mathrm{~min}$, and then the $\mathrm{pH}$ was checked and if necessary adjusted again. At this point acutely purified RGCs were resuspended in the corresponding medium and cultured for $48 \mathrm{~h}$ in $10 \% \mathrm{CO}_{2}$, after which they were imaged for survival and neurite growth quantification. Medium pH stability for up to $48 \mathrm{~h}$ was confirmed in advance by monitoring $\mathrm{pH}$ over time for each culture medium condition.

Immunocytochemistry. Primary antibodies included three anti-sAC antibodies. First, we raised a new affinity-purified rabbit anti-mouse/rat sAC antibody (Bio-mer) against amino acids KEQDKAEVIGSAIQAAC of the second catalytic domain of full-length sAC. This domain was chosen as it seemed more likely to be found in catalytically active somatic sAC isoforms (Farrell et al., 2008). Second, we used a rabbit anti-human polyclonal sAC antibody (generous gift from Dr. Weidong Geng, University of Texas, Dallas, TX; 1:800). Third, a rabbit anti-mouse polyclonal sAC antibody (Abcam Ab82854, 1:50), gave similar results and was used for cryosections in Figure $8 E$ (see below). Other primary antibodies included a mouse monoclonal anti-cAMP (Abcam ab70280, 1:100), rabbit monoclonal anti-PKA $2 \beta$ regulatory subunit (Abcam ab75996, 1:100), goat anti-Epac1 (Santa Cruz Biotechnology sc-8879, 1:100), goat antiEpac2 (Santa Cruz Biotechnology sc-26364, 1:100), rabbit polyclonal anti-Brn-3b (Abcam ab32264, 1:200), and mouse monoclonal anti-tau (Sigma T9450, 1:50). All secondary antibodies were Alexa fluorophoreconjugated (Invitrogen, 1:500).

Purified RGCs cultured in slide chamber wells or retinal cryosections were fixed for $15 \mathrm{~min}$ in $4 \%$ paraformaldehyde, washed in PBS (3 times), blocked, and permeabilized with 50\% goat serum (donkey serum for Epac staining) and $0.2 \%$ Triton X-100 for $1 \mathrm{~h}$; washed in PBS (3 times), incubated overnight with primary antibody, washed in PBS (3 times), incubated $2 \mathrm{~h}$ with secondary antibody, in some cases in the presence of fluorophore-conjugated phalloidin, washed with PBS (3 times), and mounted in Vectashield with DAPI. For paraffin sections of the Lex/ Sacytm1 KO mice and WT controls (Lexicon Genetics), deparaffination was performed with 210 min $100 \%$ xylene washes, serial hydration in $100 \%, 95 \%$, and $70 \%$ ethanol (5 min each), and antigen retrieval in citrate buffer and microwaved (30 $\mathrm{min})$. Immunostaining was performed as above without further fixation. For confocal microscopy, single optical sections $0.3-0.5 \mu \mathrm{m}$ in thickness were imaged from $30 \mu \mathrm{m}$ retinal sections or cultured RGCs using a $40 \times$ oil-immersion lens on an inverted confocal microscope (Zeiss, LSM 470). The laser power, gain, and offset values were matched between images with controls, for example between injured and uninjured retinas in Figure 8 (see below).

Western blot. Protein extracts from acutely purified postnatal RGCs lysed in NP40 buffer with protease and phosphatase inhibitors were quantified using the DC Protein Assay Kit II (Bio-Rad, catalog \#5000112). Lysates were electrophoresed and transferred to PVDF membranes with the Invitrogen NuPAGE system, blocked with 3\% BSA, incubated with primary rabbit anti-mouse/rat antibody overnight, washed three times with TBST buffer, incubated $2 \mathrm{~h}$ with secondary antibody, and developed with ECL reagent and film.

Reverse transcriptase-PCR. Purified RGC total RNA was extracted from RGC cell pellets (Qiagen RNeasy) and cDNA synthesized (I-script kit,
Bio-Rad thermocycler). For SAC cDNA sequence analysis, $2 \mu \mathrm{l}$ of cDNA (equivalent to $150 \mathrm{ng}$ of RNA) was amplified by a standard three-step protocol using Platinum TaqPCR Kit (Invitrogen) with an initial denaturation step at $94^{\circ} \mathrm{C}$ for $2 \mathrm{~min}$, following by 35 cycles of $94^{\circ} \mathrm{C}$ for $30 \mathrm{~s}$, annealing at $65^{\circ} \mathrm{C}$ for $30 \mathrm{~s}$, and extension at $68^{\circ} \mathrm{C}$ for $1 \mathrm{~min} 40 \mathrm{~s}$, followed by a final step at $68^{\circ} \mathrm{C}$ for $10 \mathrm{~min}$. The following primers were used to amplify sAC transcripts: (1) rat sAC C terminus, forward: 5'-CAT GAG TAA GGA ATG GTG GTA CTC A-3'; reverse: 5' -AGG GTT ACG TTG CCT GAT ACA ATT-3' (Pastor-Soler et al., 2003); (2) catalytic domain of the sAC brain isoform, forward: 5' GAT CGT GTT TG-3'; reverse: 5'-TGT GCA CAC AGG CAG CTT GGAT GG-3'; (3) ATPase domain, forward: 5'-TGG GAC CGT TAG CCT CCT GGG CCT TAC-3'; reverse: 5'CCC GGA TTT CCT GAG GCT GCA TGG-3'; (4) $1180 \mathrm{bp}$ fragment, forward: 5' -CCC GGA TTT CCT GAG GCT GCA TGG-3; reverse: 5'-CCC GGA TTT CCT GAG GCT GCA TGG-3'. PCR products were visualized by agarose gel electrophoresis and ethidium bromide staining, and extracted from the gel by QIAquick Gel Extraction Kit (Qiagen). The 1180 bp fragment was cloned using the T-A Cloning Kit (Invitrogen) by the manufacturer's directions. A total of 5 clones were randomly selected and the nucleotide sequence of the inserted fragment from each clone was determined by DNA sequencing (Genewiz). Visualization of alignment to the genome (Rattus norvegicus) and exons was done using Genomatix Genome Browser.

Intracellular cAMP quantification. Cell pellets of postnatal rat RGCs were incubated $2 \mathrm{~h}$ in medium with or without bicarbonate and with or without forskolin, centrifuged, and flash frozen in liquid nitrogen. cAMP was quantified with the EIA ELISA Kit (Cayman Chemical) using the acetylation method according to their instructions.

Electrical stimulation. Cultured RGCs were electrically stimulated in chamber slides (Thermo Scientific Nunc Lab-Tek II Chamber Slide System, \#154534) modified by perforating the chamber wall with two heated stainless steel needles at the wall slide interface. Wells were coated with mouse laminin ( $1 \mu \mathrm{g} / \mathrm{ml}$; Invitrogen) for $3 \mathrm{~h}$, before plating $10-15,000$ RGCs per well in growth medium. After $2 \mathrm{~h}$ to allow the cells to settle, time lapse images of random fields were taken in a Zeiss $200 \mathrm{~m}$ inverted microscope for quantifying baseline cell survival (thereafter set at $100 \%$ for normalization). Two monopolar $22 \mathrm{~mm}$ stainless steel disposable EEG needle electrodes (Chalgren Enterprises) were inserted in parallel through the premade perforations, to the opposite wall in close contact with the glass slide. Cells were electrically stimulated with a pulse generator (Berkeley Nucleonics 575-4C), modified with $300 \Omega$ resistors in series and $300 \Omega$ resistors in parallel to decrease any basal output present during resting conditions. Cells were stimulated from 2 to $28 \mathrm{~h}$ by $2.5 \mathrm{~V}$, $4 \mathrm{~mA}, 10 \mathrm{~Hz}$ pulse trains every $200 \mathrm{~s}$. Each train was composed of 7-10 1 ms pulses, actual output $2.5 \mathrm{~V}, 4 \mathrm{~mA}$ at $10 \mathrm{~Hz}$. After electrical stimulation, the electrodes were removed and the same fields imaged at $2 \mathrm{~h}$ were reimaged. Live cells were labeled with the vital dye calcein-AM (AnaSpec, \#89201). Survival was quantified as the percentage of cells alive at the end of the experiment normalized to the $2 \mathrm{~h}$ time point. Axons were defined morphologically as thin, nontapering neurites extending at least one cell body diameter. Average axon growth per cell was measured as total axon growth divided by the number of surviving cells in each image.

siRNA electroporation. Postnatal rat RGCs $(200,000)$ were electroporated immediately after purification either with control (scrambled) siRNA or anti-sAC siRNA (Sigma, PDSIRNA sasi_Rn01_00054849 and PDSIRNA sasi_Rn01_00054853), 1 nmol per electroporation reaction in $27 \mu \mathrm{l}$ of electroporation buffer using the Amaxa Nucleofector II electroporator with the neuron small cell number program number 1 , and an optimized electroporation buffer (aqueous solution containing $25 \mathrm{~mm}$ sodium pyruvate, $0.3 \%$ isobutyric acid, $50 \mu \mathrm{g} / \mathrm{ml}$ bovine serum albumin, $1 \mathrm{~mm}$ magnesium sulfate and $100 \mathrm{~mm}$ dipotassium phosphate). RGCs were cultured in slide chambers. Images of 10-12 random fields per condition were taken at 2 and $48 \mathrm{~h}$ to quantify cell survival and axon growth. Data were analyzed from at least two independent experiments.

Intravitreal injections. Saline $(3 \mu \mathrm{l})$ with DMSO (control) or $66 \mu \mathrm{M}$ $\mathrm{KH} 7$ in DMSO was injected intravitreally into the left eye of anesthetized adult Sprague Dawley female rats, taking care to avoid the lens and to release elevated eye pressures by anterior chamber paracentesis. Animals were euthanized $5 \mathrm{~d}$ postinjection, and eyes were surgically removed, 

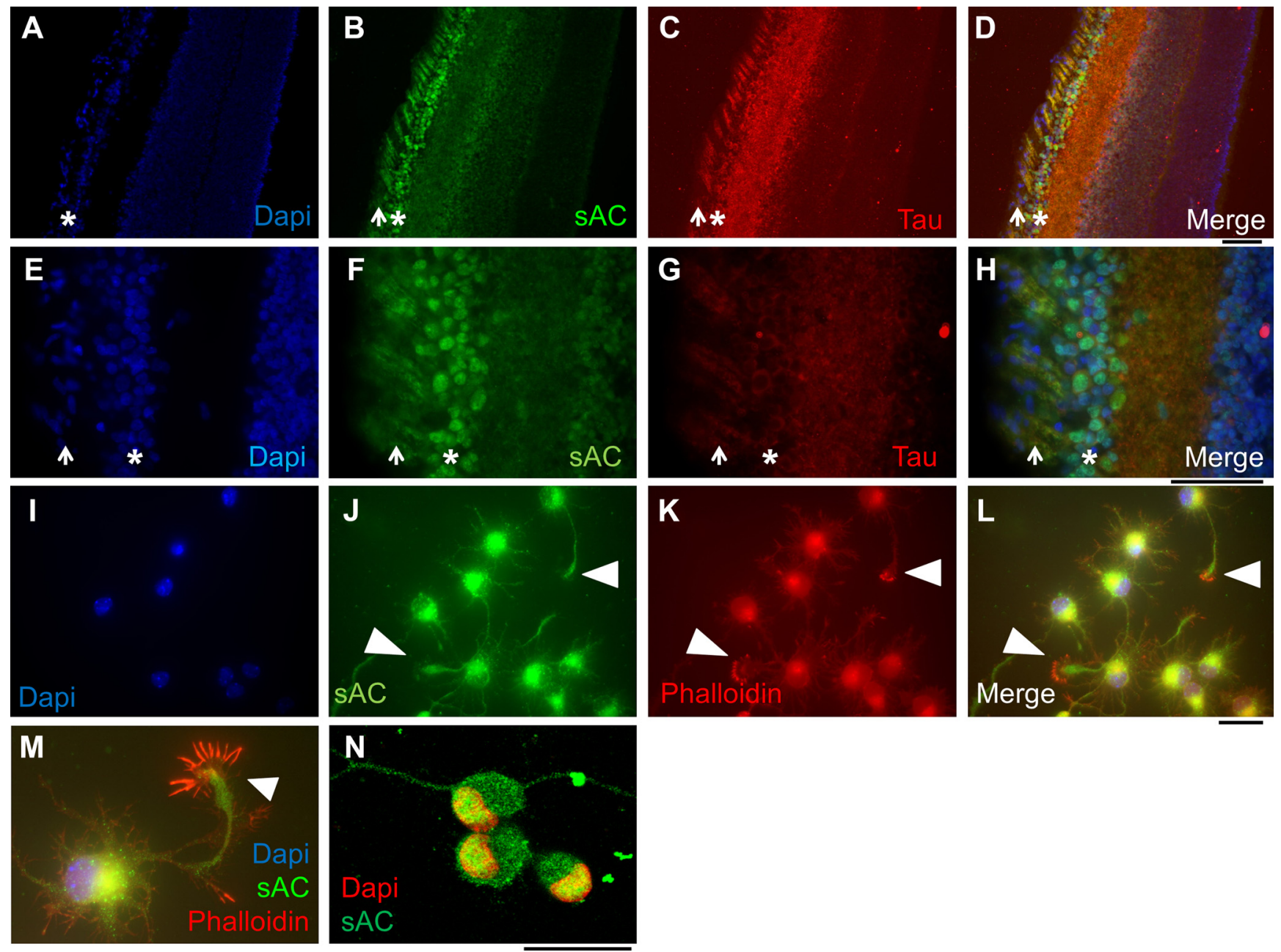

Figure 1. $\quad \mathrm{SAC}$ is expressed in $\mathrm{RGCS} . \boldsymbol{A}-\boldsymbol{H}, \mathrm{sAC}$ expression $(20 \times, \boldsymbol{A}-\boldsymbol{D} ; 63 \times, \boldsymbol{E}-\boldsymbol{H})$ was detected by immunostaining adult rat retinal frozen sections with anti-human $\mathrm{SAC}$ ( $g r e e n)$ and anti-tau (red); nuclei counterstained with DAPI (blue). SAC was detected in the ganglion cell layer (asterisks) and RGC axons in the nerve fiber layer (arrows). I- L, In P3 RGCs after $1 \mathrm{~d}$ in vitro, SAC was detected with rabbit anti-mouse/rat $\mathrm{SAC}$ antibody (green); counterstaining highlights cytoplasm and neurites (phalloidin, red) and nuclei (DAPI, blue). sAC immunoreactivity was detected in the nucleus, cytoplasm, and axons, and in the central but not the peripheral growth cone domain (arrowheads). $\boldsymbol{M}$, Another example as in $\boldsymbol{I}-\boldsymbol{L}$ at higher magnification. $\boldsymbol{N}, \mathrm{sAC}(63 \times$ magnification, green) was detected in the nucleus by confocal imaging (red; colocalization in yellow). Scale bars, $25 \mu \mathrm{m}$.

fixed in $4 \%$ paraformaldehyde, embedded in O.C.T, and frozen in liquid nitrogen for cryosectioning. Sections $(12 \mu \mathrm{m})$ were immunostained as described above for purified RGCs, and all Brn3/cAMP-positive cells in the retinal ganglion cell layer were counted every fifth section. RGCs were counted by hand by a lab member masked to the experimental conditions.

Optic nerve crush. Optic nerve crush was performed as described previously (Usher et al., 2010). Animals were euthanized $6 \mathrm{~d}$ after injury and PFA-fixed eyes were cryosectioned to $10 \mu \mathrm{m}$, immunostained, and analyzed using confocal microscopy.

\section{Results}

Retinal ganglion cells express soluble adenylyl cyclase

First we asked whether sAC is expressed in mammalian RGCs by staining sagittal frozen sections of the adult rat retina (Fig. $1 A-H$ ) with antiserum against the $\mathrm{N}$ terminus (exon 5) of the human sAC (generous gift from Dr. Weidong Geng). Stronger staining was detected in the ganglion cell layer (GCL) compared with other layers in the retina (Fig. $1 A-D$ ). sAC protein was detected in the nuclei and cytoplasm of GCL cells, including RGC dendrites in the inner plexiform layer, and RGC axons in the nerve fiber layer (Fig. $1 A-H$ ), indicating that among the cells in the retina, sAC is more highly expressed in adult mammalian RGCs.
We raised a second, rabbit anti-mouse/rat affinity-purified antibody (see Materials and Methods) against a peptide fragment from the catalytic domain of the rodent somatic (brain) sAC isoform (Farrell et al., 2008). This anti-sAC antibody's specificity was validated by immunostaining the Lex/Sacytm $1 \mathrm{KO}$ mouse, in which full-length, testis-specific sAC isoforms are not expressed, but truncated isoforms are still detected somatically (Farrell et al., 2008). In paraffin sections (gift from Lexicon Genetics), the new antibody stained nuclei of WT but not sACdeficient $\mathrm{KO}$ testis (Fig. $2 A-D$ ), demonstrating specificity. However, with this antibody sAC was detected in all retinal layers in both WT and Lex/Sacytm $1 \mathrm{KO}$ (Fig. 2E-H). Differences in retinal subcellular localization (compare Fig. $1 B, F$ ) could suggest that different isoforms are present in different retinal layers, or be an artifact of the different fixations (Dapson, 2007). Thus, by two distinct antibodies, RGCs in vivo express one or more sAC isoforms containing the second catalytic domain even after fulllength sAC knock-out.

Immunostaining with rabbit anti-mouse/rat sAC antibody also confirmed sAC expression in purified mouse RGCs. sAC was detected in nuclei, soma, and neurites of all RGCs in culture (Fig. $1 I-N)$. The same staining pattern was observed in RGCs using a 

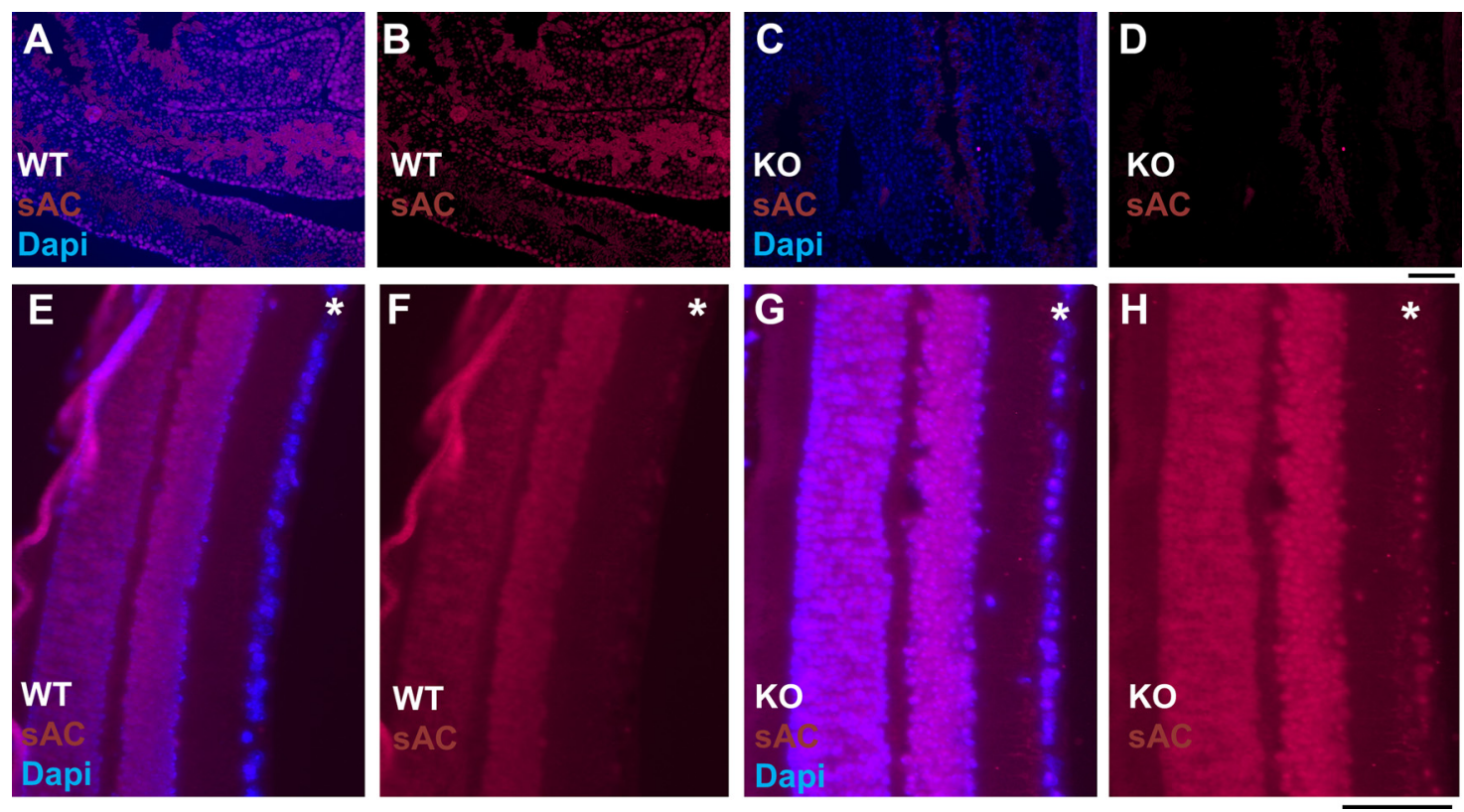

Figure 2. Validated anti-sAC antibody detects $S A C$ in retinal cells even in Sacytm1Lex/Sacytm1Lex K0 mice. $\boldsymbol{A}-\boldsymbol{D}$, Immunostaining with rabbit anti-mouse/rat $s A C$ detected $s A C$ in the nuclei of WT murine testis $(\boldsymbol{A}, \boldsymbol{B})$ but not in the Sacytm1Lex/Sacytm1Lex KO $(\boldsymbol{C}, \boldsymbol{D})$, confirming antibody specificity. $\boldsymbol{E}-\boldsymbol{H}$, In contrast, in the retina anti-sAC was detected in nuclei in all layers including the ganglion cell layer (asterisks) in both WT $(\boldsymbol{E}, \boldsymbol{F})$ and $K 0$ retinas $(\boldsymbol{G}, \boldsymbol{H})$ indicating that one or more sAC isoforms not deleted in the $\mathrm{K} 0$ are expressed in retinal cells including RGCs. Scale bars, $25 \mu \mathrm{m}$.

third, commercially available rabbit anti-mouse sAC antibody (see Materials and Methods). We also detected cAMP, the regulatory subunit of PKA 2B, Epac-1 and Epac-2 immunoreactivity in RGC nuclei, as well as immunoreactivity outside of RGC nuclei in vitro (Fig. 3). These data support the presence of a complete intranuclear cAMP signaling pathway that may include sAC itself (Zippin et al., 2004), but we did not pursue nuclear versus non-nuclear-specific roles for sAC further in this series of experiments.

Next we characterized sAC isoform expression in RGCs. By reverse transcriptase (RT)-PCR we amplified four different fragments of the sAC transcript: three short fragments corresponding to the catalytic domain, predicted ATPase domain, and C terminus (Fig. 4A), and an $1180 \mathrm{bp}$ fragment spanning the alternative (somatic) start codon (Farrell et al., 2008) through the ATPase domain (Fig. 4B). This last fragment did not correspond to the expected product size (according to GenBank accession number NM_021684.1); however, complete sequencing of the $1180 \mathrm{bp}$ fragment as well as sequencing of the other RT-PCR three shorter fragments confirmed sAC's identity in all cases, and indicate RGC expression of a Sac splicing isoform not previously described (Fig. 4E), lacking 192 bp between residue 1925 and 2117 of the mRNA. In addition, siRNA-mediated sAC knockdown almost completely eliminated endogenous expression of this $1180 \mathrm{bp}$ fragment (Fig. $4 B, D$ ), confirming both RT-PCR and siRNA specificity and efficacy. Smaller fragments were differentially reduced in expression as measured nonquantitative RT-PCR densitometry (Fig. $4 A, C$ ), which again may indicate expression of smaller $\mathrm{N}$ - and C-terminal isoforms not well targeted by the siRNAs (Fig. $4 E$ ); the presence or impact of such isoforms was not investigated further. Finally, our new anti-sAC catalytic domain antibody detected only one band on Western blot at $50 \mathrm{kDa}$ in purified postnatal rat RGC protein extracts (Fig. $4 F$ ), suggesting that in postnatal RGCs, either one of the sAC isoforms is more abundant or other transcripts are translationally silenced. It is also possible that other isoforms are not detected by this antibody, or that multiple isoforms do not differ significantly in size.
Finally, we assayed for sAC enzymatic activity by ELISA in response to the physiologic sAC-specific agonist bicarbonate, which does not activate other ACs (Jaiswal and Conti, 2001; Geng et al., 2005) (in contrast to calcium, which also activates AC1 and -8). Carefully controlling for $\mathrm{pH}$ (see Materials and Methods), we detected a significant bicarbonate-induced increase in intracellular cAMP, which was enhanced by the tmAC-specific activator forskolin (Fig. 4G). Thus RGCs express sAC protein in vitro and in vivo, sAC protein is distributed throughout the cell, and bicarbonate-induced enzymatic sAC activity is present in RGCs, and is additive to forskolin-induced tmAC activity in elevating cAMP.

\section{Activating sAC promotes RGC survival and axon growth}

We next asked whether sAC activation mediates RGC survival and axon growth. Survival was unaffected by bicarbonate (Fig. $5 A-E$ ). In contrast, bicarbonate increased RGC axon growth significantly in a dose-dependent manner (Fig. $5 F$ ). Consistent with the additive effect of SAC and tmAC activation on cAMP levels (Fig. $4 D$ ), the bicarbonate-induced increase in axon growth was further enhanced by forskolin (Fig. $5 F$ ). These results indicate that the sAC activator bicarbonate promotes axon growth in a pathway separate from and additive to the tmAC activator forskolin.

\section{Inhibiting sAC decreases RGC survival and axon growth}

Two noncompetitive sAC inhibitors, KH7 and 2-hydroxyestradiol (2HE) were examined to determine whether inhibiting sAC affects RGC survival or axon growth. Although both bind to sAC's catalytic domain, they are structurally and mechanistically distinct (Schlicker et al., 2008). Specifically, $2 \mathrm{HE}$ belongs to the family of catechol estrogens; using crystallographic modeling of sAC in the algae Spirulina platensis (Steegborn et al., 2005b), and from bacteria (Steegborn et al., 2005a) and Pseudomonas aeruginosa (Topal et al., 2012), the catechol in $2 \mathrm{HE}$ chelates the catalytic divalent ion calcium or magnesium. Although the steroid scaffold has the potential to bind to a pocket in all class III cyclases including membrane-bound 

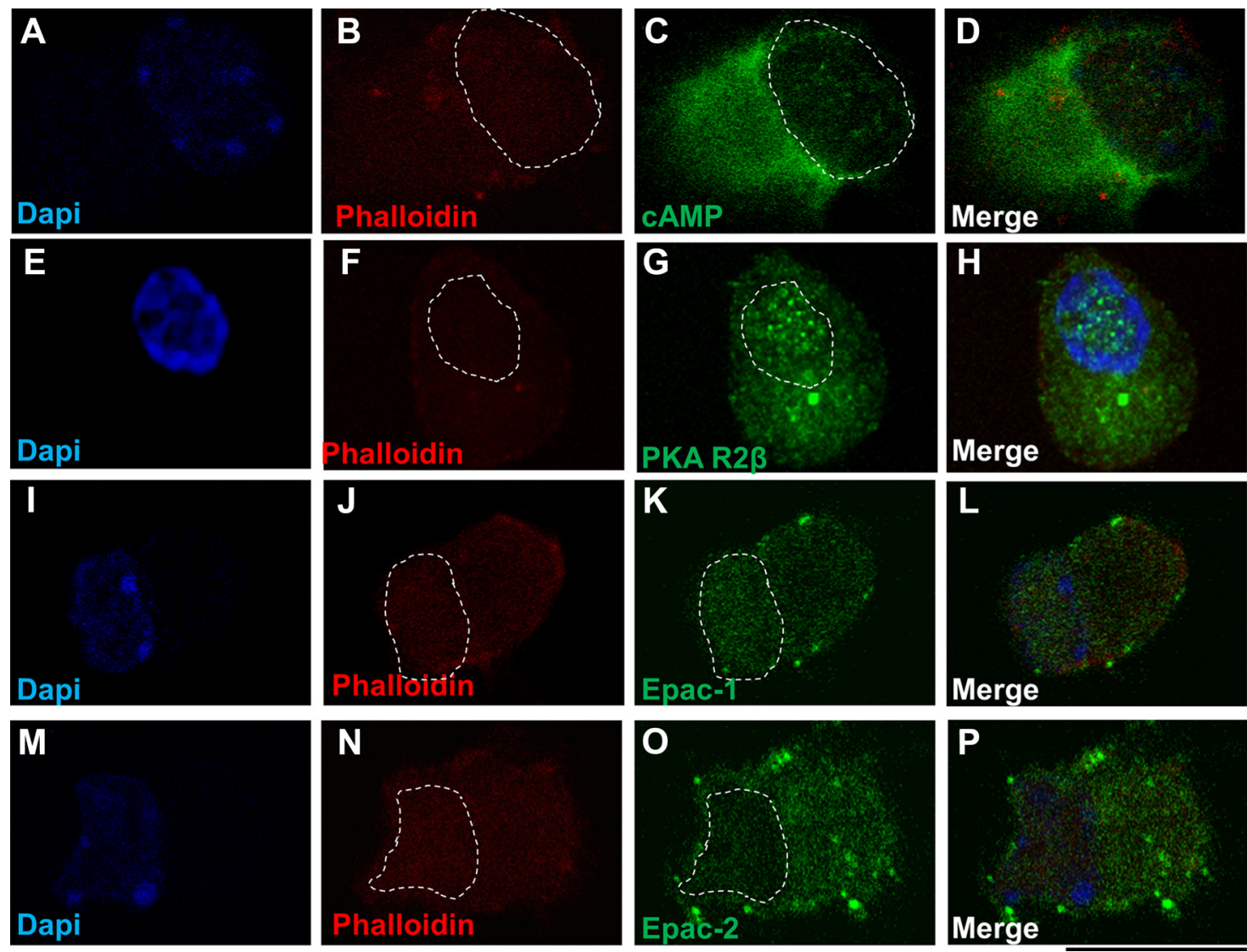

Figure 3. CAMP, PKA regulatory subunit $2 B$, Epac-2 and Epac- 1 are detected in intracellular compartments including RGC nuclei. $A-P$, Confocal imaging of dissociated P5 RGCs immunostained as marked (green), and counterstained with phalloidin (red) and DAPI (blue), to highlight the cells and their nuclei (dotted outlines shown across images), respectively. Scale bar (in $\boldsymbol{P}$ ) $\boldsymbol{A}-\boldsymbol{P}, 25 \mu \mathrm{m}$.

ACs, the compound is potent and specific for sAC inhibition at the concentrations used in this paper $(<50 \mu \mathrm{M})$ and in most of the sAC literature (Steegborn et al., 2005b). In contrast, the noncompetitive inhibitor KH7 does not have the catechol moiety, and although its exact mechanism of action is not known, it is specific for sAC-like ACs even a higher concentrations ( $>100 \mu \mathrm{M})$ (Hess et al., 2005).

We found a dose-dependent decrease in RGC axon growth but no effect on survival at low concentrations with both compounds (Fig. 6A-D). 2HE applied at $24 \mathrm{~h}$ after RGCs had extended axons, induced axon retraction and fragmentation (Fig. $6 \mathrm{~A})$. KH7 applied at the time of plating prevented axon growth (Fig. 6C). At the higher doses usually reported in the literature (10-50 $\mu \mathrm{M}$ ) (Wang et al., 2005; Li et al., 2011), both sAC inhibitors significantly decreased RGC survival (Fig. $6 E-G$ ). We also noticed that after longer periods of axon growth inhibition by the lower concentrations of sAC inhibitors in vitro, RGCs did die. Such phenomena are reported to happen in other neurodegenerative diseases like ALS, and after axon injury and target-derived trophic factor deprivation, as after optic nerve injury. The results with these two specific inhibitors strongly suggest that sAC is necessary for survival and axon growth, and that axon growth and integrity are more sensitive to sAC inhibition than cell survival, consistent with the results observed with sAC activation in Figure 5.

We next asked whether knocking down sAC using siRNAs similarly affects RGC survival or axon growth. Anti-sAC siRNA, targeted to sAC's second catalytic domain from the full-length sAC (which is the only catalytic domain in somatic sAC) reduced both survival and axon growth. RGC axons grew for the first $24 \mathrm{~h}$; however, at $48 \mathrm{~h}$, anti-sAC siRNA-treated RGCs exhibited patchy axon growth patterns and cell body fragmentation in concert with decreased survival and axon growth (Fig. $6 H, I$ ). siRNA transfection efficiency was $\sim 70 \%$, but we only analyzed cells cotransfected with a GFP reporter plasmid. Thus, inhibiting sAC, both pharmacologically and with anti-sAC siRNA, indicates sAC is necessary for RGC survival and axon growth in vitro, although axon growth ability depends more sensitively on sAC activity.

\section{RGC survival and axon growth are minimally affected after} inhibiting or knocking out tmACs

To further investigate the differences between tmAC and sAC effects on RGCs survival and axon growth, we investigated pharmacological tmAC inhibitors and AC1/AC8 double $\mathrm{KO}$ mice. We found that tmAC inhibition with either SQ22536 (Fig. 7A, B) or 2,5-dideoxyadenosine (Fig. $7 C$ ) did not significantly decrease RGC survival or axon growth, compared with the inhibitory effect of sAC inhibition, when cultured in growth factor- and forskolin-containing medium known to promote RGC survival and axon growth (Meyer-Franke et al., 1995; Goldberg et al., 2002). The competitive PKA inhibitor H89, however, decreased RGC survival and neurite growth (Fig. $7 D, E$ ), consistent with prior data (Goldberg et al., 2002). These results suggest that tmACs may be less important than sAC in PKA-mediated RGC survival and axon growth. We tested this hypothesis by purifying and culturing neonatal RGCs from AC1/AC8 double $\mathrm{KO}$ mice. Neither survival nor axon growth were altered in AC1/AC8 dou- 


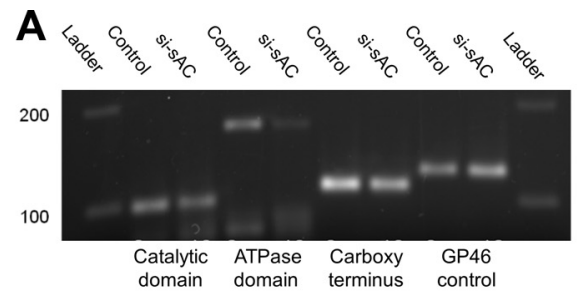

C

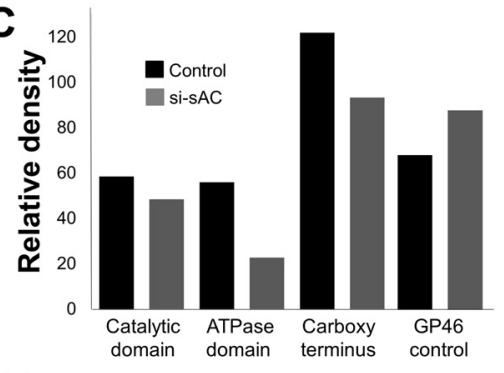

E
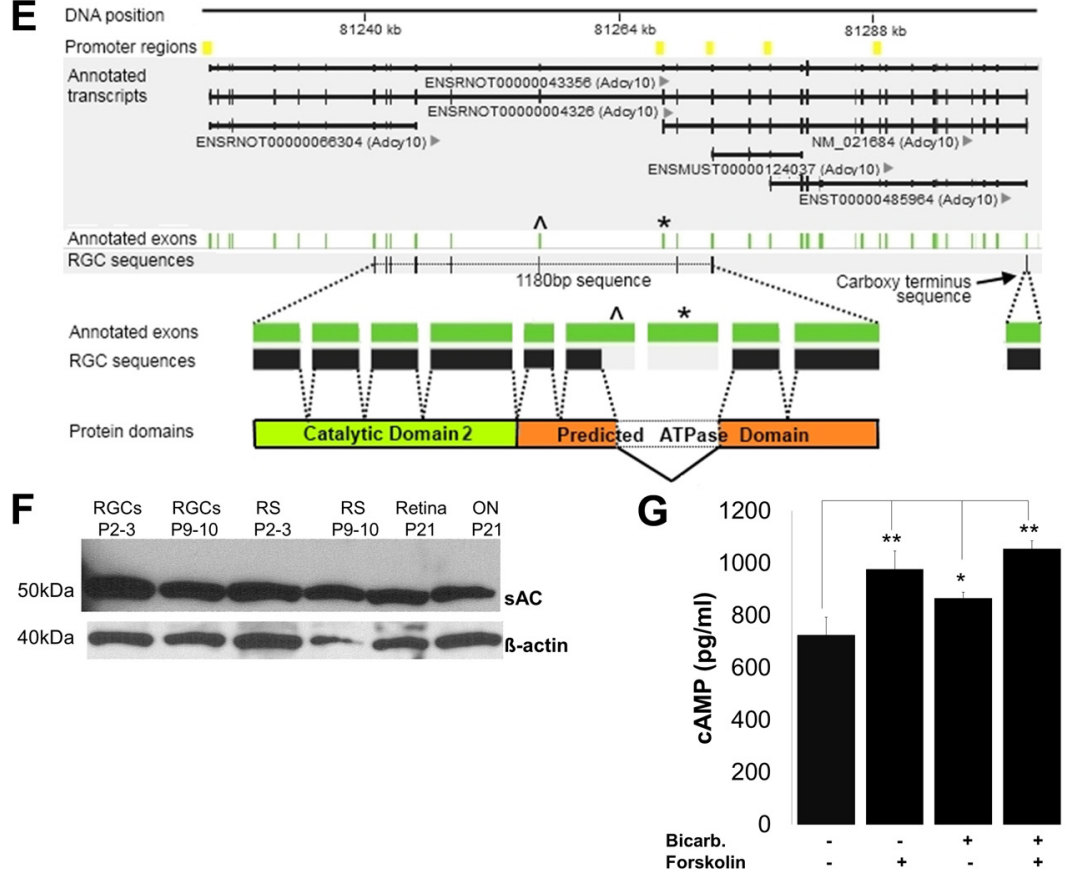

Figure 4. $\quad$ sAC is expressed and active in RGCS.A, RT-PCR in scrambled siRNA-treated (control) and anti-sAC siRNA-treated (si-sAC) RGCS using primers corresponding to the somatic sAC catalytic domain (100 bp), the putative ATPase domain (174bp), and the C terminus of the full-length isoform (111 bp), as well as the collagen binding protein GP46 (125 bp) as a control. RNA was collected 48 hafter electroporation. Qualitative reduction in SACPCR products was detected in the sAC siRNA-treated RGCS. B, RT-PCR detected a longer fragment of $\sim 1180 \mathrm{bp}$ between the alternative start codon of somatic $\mathrm{AC}$ and the putative ATPase domain which is eliminated $48 \mathrm{~h}$ after anti-sAC siRNA electroporation. Ribosomal protein $18 S$ (110 bp) was used as a control. All fragments were confirmed to be sAC by sequencing ( $N$ o RT, no reverse transcriptase control). C, Relative densitometry (NIH ImageJ software) was performed on the gel in $A$. A clear reduction in PCR band density can be seen in all anti-sAC siRNA-treated samples compared with untreated, except for the GP46 control. D, Replicate of RT-PCR amplification of the $1180 \mathrm{bp}$ fragment confirms decreased expression of the transcript in anti-sAC siRNA-treated RGCS. $\boldsymbol{E}$, The $1180 \mathrm{bp}$ fragment cloning and sequencing demonstrates a Sac isoform with 6 of 8 exons aligning to annotated sAC exons. The RGC isoform was missing 192 bp between residues 1925 to 2117 of the predicted ATPase domain of the full-length SAC mRNA; the exon labeled $\wedge$ is truncated and the following exon, labeled *, is skipped. These two exons are present in every annotated transcript that contains exons from the $1180 \mathrm{bp}$ sequence flanking the truncated and missing exons, suggesting a novel sAC isoform. $\boldsymbol{F}$, By Western blot, mouse/rat-sAC detected a single band $\sim 50 \mathrm{kDa}$ in RGCs at different postnatal ages (RGCS), macrophages-depleted retinal suspensions (RS), and whole retina or optic nerves (ON) as indicated. Anti- $\beta$-actin was the loading control. G, cAMP was quantified by triplicate ELISA for P8 RGCs after a $2 \mathrm{~h}$ incubation with or withoutbicarbonate and/or forskolin as marked (+/-) Errorbars indicateSD;ANOVA and Tukey'sposthoctest, ${ }^{*} p<0.05,{ }^{* *} p<0.01$.

ble KO RGCs, except for a small forskolin-mediated increase in survival lost in AC1/AC8 double KO RGCs that may be attributable to loss of activation of these two tmACs with forskolin (Fig. $7 F-I)$. Thus, both pharmacologic inhibition and AC1/AC8 knock-out suggest tmAC activity, at least for the $\mathrm{Ca}^{2+}$-activated
tmACs-1 and -8 , is less important than sAC activity for RGC survival and axon growth.

\section{Electrical stimulation requires $\mathrm{sAC}$ activity to promote survival}

Prosurvival and regenerative effects of electrical stimulation in RGCs require cAMP (Goldberg et al., 2002), and depolarization-induced molecular oscillations in cAMP are mediated in part by non-tmACs in RGCs (Dunn et al., 2009). Therefore, we tested the effect of electrical stimulation in the presence of the tmAC activator forskolin and the sAC activator bicarbonate (25 mM in Neurobasal). Electrical stimulation did not increase RGC axon growth above the already high level obtained with growth factors and forskolin (Fig. 8B), but significantly increased RGC survival even in the presence of forskolin (Fig. 8A). Inhibiting sAC with 2-HE abrogated the added prosurvival effect of electrical stimulation, suggesting that cAMP produced by sAC, not tmAC, is necessary to promote RGC survival with electrical stimulation. Moreover, these data indicate that electrical activity and tmAC activators may work either alone or in combination toward increasing RGC survival and/or axon growth, and that sAC activation by bicarbonate and by electrical stimulation (which may act through calcium entry) themselves collaborate to increase RGC survival (see Discussion below).

\section{sAC activity in vivo is necessary for RGC survival}

We next asked whether RGC survival depends on sAC activity in vivo. Prior data showed the electrical activity-cAMPsurvival pathway is active in the uninjured adult retina, but after axon injury this signaling pathway is decreased (Shen et al., 1999). Thus, we hypothesized sAC plays a role in survival signaling in the normal adult RGC. Injecting the sAC inhibitor $\mathrm{KH} 7$ significantly reduced RGC survival in adult rat retinas after $5 \mathrm{~d}$ in vivo compared with DMSO control (Fig. $8 C, D$ ). In contrast, we were unable to detect increased RGC death after optic nerve injury using sAC inhibitors or anti-sACsiRNA injected either intravitreally or at the injured optic nerve suggesting the hypothesis that SAC activation may already be reduced after optic nerve injury. Indeed when we assayed for sAC protein expression at $6 \mathrm{~d}$ after optic nerve injury, before significant RGC death commences, there was a reduction in $\mathrm{sAC}$ immunoreactivity in the nerve fiber layer (Fig. $8 E$ ). Thus, sAC activity is necessary for RGC survival under physiological conditions, and there may be a reduction in $\mathrm{AAC}$ after optic nerve injury. 


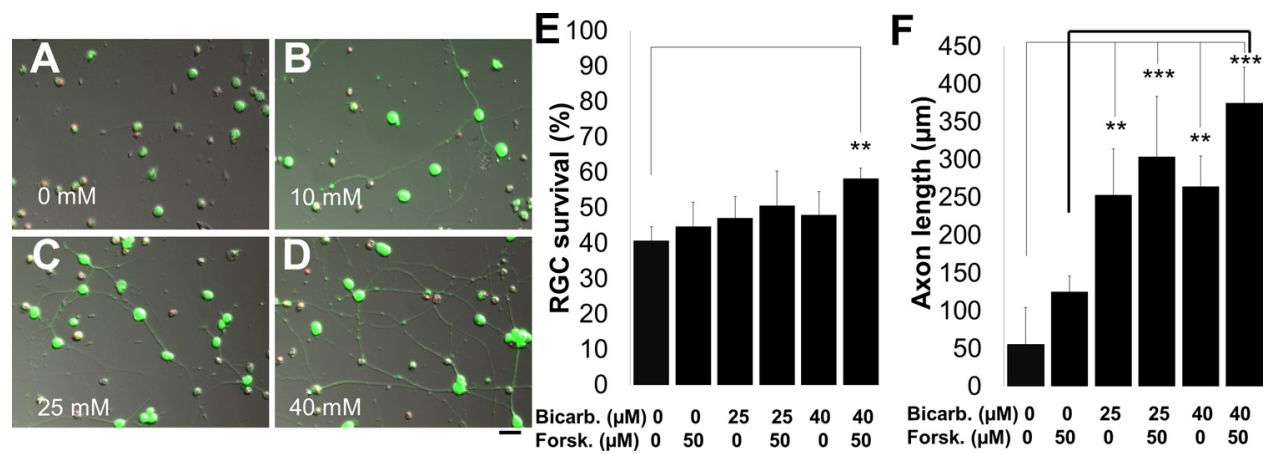

Figure 5. Bicarbonate stimulates $\mathrm{RGC}$ survival and growth. $\boldsymbol{A}-\boldsymbol{D}$, Representative pictures of $\mathrm{P} 9 \mathrm{RGCS} 48 \mathrm{~h}$ in culture with or without bicarbonate (concentrations as marked), labeled with the live cell indicator calcein green. Notice the robust axon growth in $40 \mathrm{~mm}$ bicarbonate compared with the absence of growth without bicarbonate (control). Scale bar, $25 \mu \mathrm{m}$. $\boldsymbol{E}$, The physiologic sAC activator bicarbonate and the tmAC activator forskolin together slightly promote survival in a medium rich in growth factors. $\boldsymbol{F}$, Bicarbonate and forskolin promote RGC axon growth. Error bars, SD; $n \geq 100$ neurons per condition, ANOVA and Tukey's post hoc test, ${ }^{* *} p<0.01,{ }^{* *} p<0.001$; representative data from repeated experiments shown for $\boldsymbol{E}, \boldsymbol{F}$.

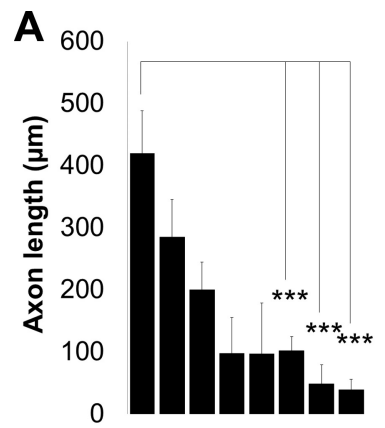

2HE ( $\mu$ M) $01223 \quad 451015$

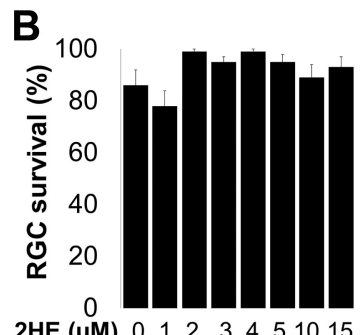

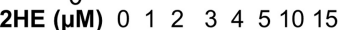

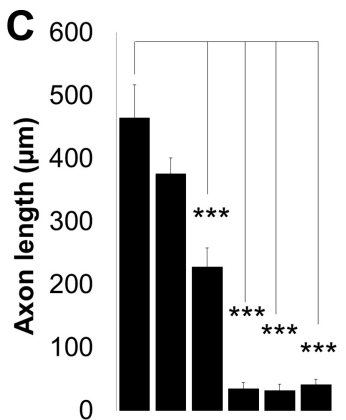

KH7 ( $\mu$ M) $0 \begin{array}{llllll}0 & 1 & 2 & 3 & 5 & 10\end{array}$

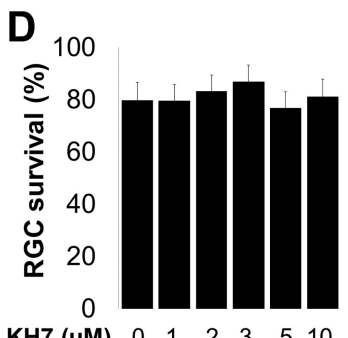

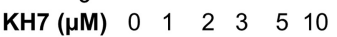
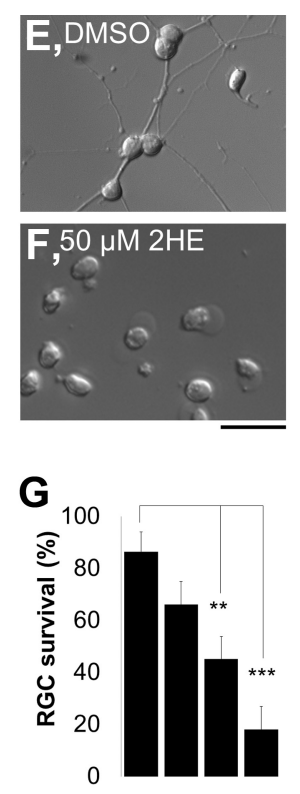

2HE ( $\mu$ M) 0203550

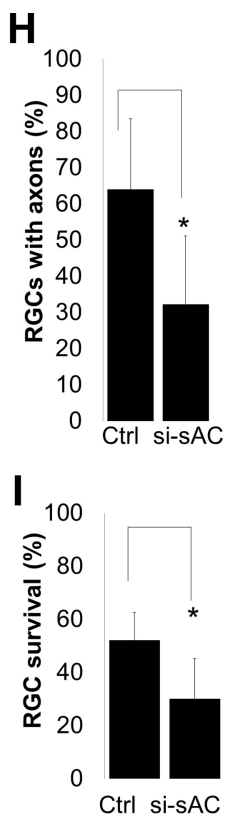

Figure 6. Inhibiting $\mathrm{SAC}$ pharmacologically or with siRNA decreases $\mathrm{RGC}$ axon growth and survival. $A, B$, Exposing P5 RGCs to low micromolar concentrations of the pharmacological sAC inhibitor 2-HE from 24 to $48 \mathrm{~h}$ in vitro decreased axon growth and induces axon retraction without any effect on RGC survival. C, D, The pharmacological sAC inhibitor KH7 applied from 2 to $24 \mathrm{~h} \mathrm{inhibited} \mathrm{axon}$ growth without affecting RGC survival. $\boldsymbol{E}$, $\boldsymbol{F}$, Representative images of RGCs cultured in DMSO $(\boldsymbol{E})$ or $50 \mu \mathrm{m} 2 \mathrm{HE}(\boldsymbol{F})$ for $24 \mathrm{~h}$, demonstrating axon growth inhibition. $\boldsymbol{G}$, sAC inhibition with higher concentrations of 2-HE decreased P5 RGC survival in a dose-dependent manner. Error bars, SD; $n \geq 100$ neurons per condition, ANOVA and Bonferroni's posthoctest, ${ }^{*} p<0.05,{ }^{* *} p<0.01,{ }^{* * *} p<$ 0.001; representative data from repeated experiments shown. $\boldsymbol{H}, \boldsymbol{I}$, Anti-sAC siRNA decreased P3 RGC axon growth $(\boldsymbol{H})$ and survival $(\boldsymbol{I})$ measured $48 \mathrm{~h}$ after electroporation compared with electroporation with a scrambled siRNA control. Error bars, $\mathrm{SD} ; t$ test, $\left.{ }^{*} p<0.05\right)$.

\section{Discussion}

Here, we show sAC is expressed in RGCs, sAC activation elevates cAMP in RGCs and promotes RGC survival and axon growth, and sAC is necessary for RGCs' survival and axon growth response to electrical activity in vitro, and for RGC survival in vivo. The second messenger cAMP plays diverse roles in neuronal physiology including neuronal survival and axon growth. Numerous tmACs are implicated in elevating cAMP in response to diverse stimuli, but here we expand on such prior work to include the specific identification of sAC as a key player in cAMP signaling in these CNS neurons.

\section{Elevating cAMP promotes survival and axon growth}

Interestingly, RGC survival and axon growth were minimally affected by tmAC inhibitors or in the AC1/AC8 KO. This contrasts with prior data in which RGCs were sensitive to trophic stimulation promoted by tmAC activation by forskolin (Meyer-Franke et al., 1995; Goldberg et al., 2002). However, RGC survival, calcium entry, and cAMP elevation were not affected in the AC1/AC8 KO (Dunn et al., 2009). Indeed, to what extent functions attributed to tmACs are actually the product of sAC activity, or how tmACs and sAC may interact or synergize is unknown. cAMP enhances neuronal survival and axon growth through diverse mechanisms, including increasing responsiveness to growth factors (Meyer-Franke et al., 1998; Goldberg et al., 2002; Stessin et al., 2006), regulating axon growth (Wu et al., 2006; Murray and Shewan, 2008), polarity (Yamada et al., 2005) and guidance (Song et al., 1997; Shelly et al., 2010), and modulating neuronal excitability and both regenerative and prosurvival responses to electrical activity or other signals (Meyer-Franke 

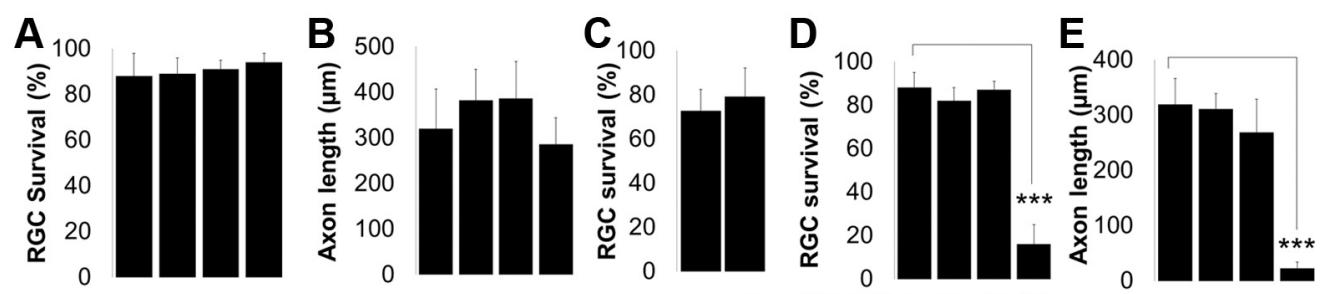

SQ ( $\mu \mathrm{M}) \quad 0300500800$

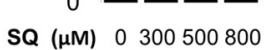

ddA $(\mu \mathrm{M}) 0200$

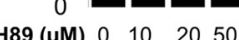

H89 (uM) $0 \quad 1020 \quad 50$
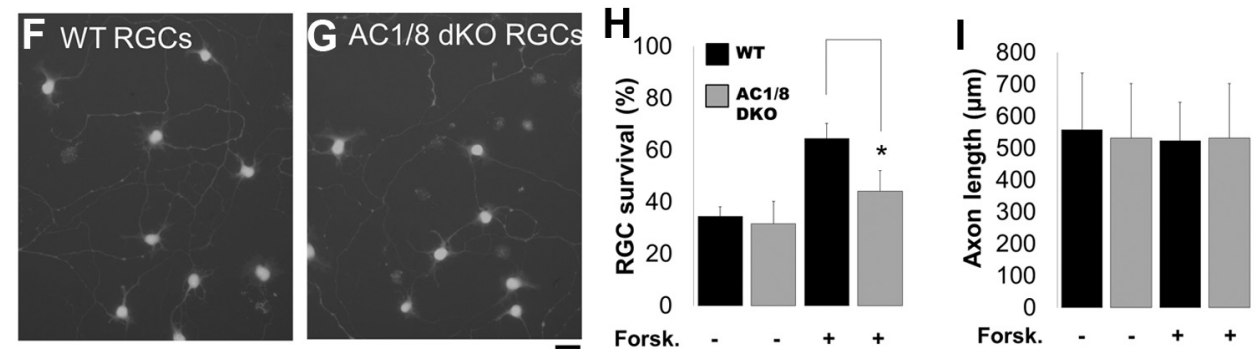

Figure 7. PKA but not tmAC activity is necessary for RGC survival and axon growth. $A, B, R G C$ survival and axon growth were unaffected by the tmAC inhibitor SQ22586. C, RGC survival was unaffected by the tmAC inhibitor 25 -ddAdo $(200 \mu \mathrm{M})$ after $48 \mathrm{~h}$ in vitro. $(N=2$; error bars, SD). $\boldsymbol{D}, \boldsymbol{E}$, The PKA inhibitor $\mathrm{H} 89$ decreased RGC survival and axon growth in the presence of growth factors and forskolin $(N=3) . F, G$, Representative images of WT $(\boldsymbol{F})$ and AC1/8 double KO RGCS $(\boldsymbol{G})$ after $24 \mathrm{~h}$ cultured in growth medium with $25 \mu \mathrm{m}$ bicarbonate and $5 \mu \mathrm{m}$ forskolin. Note the similarly healthy appearance and abundant axon growth in both sets. Scale bar, $25 \mu \mathrm{m}$. $\boldsymbol{H}$, Baseline RGC survival from the AC1/AC8 double KO mouse was not different from WT control RGCs. Only WT RGC survival increased significantly in response to forskolin. I, At $24 \mathrm{~h}$, axon growth in WT and sAC KO mice was similar, independent of forskolin (error bars SD, ANOVA and Bonferroni's posthoc test. ${ }^{*} p<$ $0.05,{ }^{* * *} p<0.001$; representative data from repeated experiments shown.
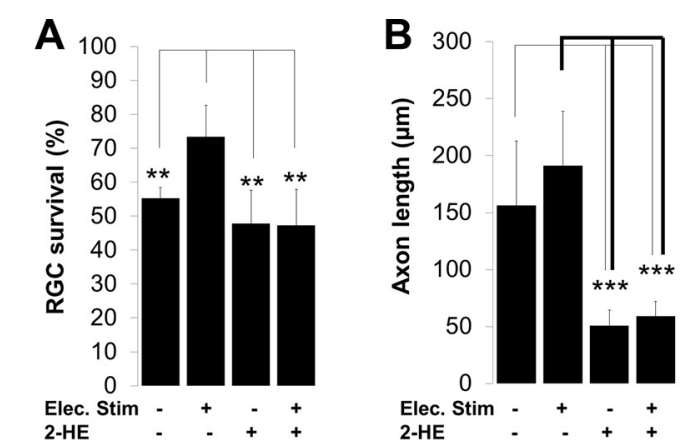

\section{E}
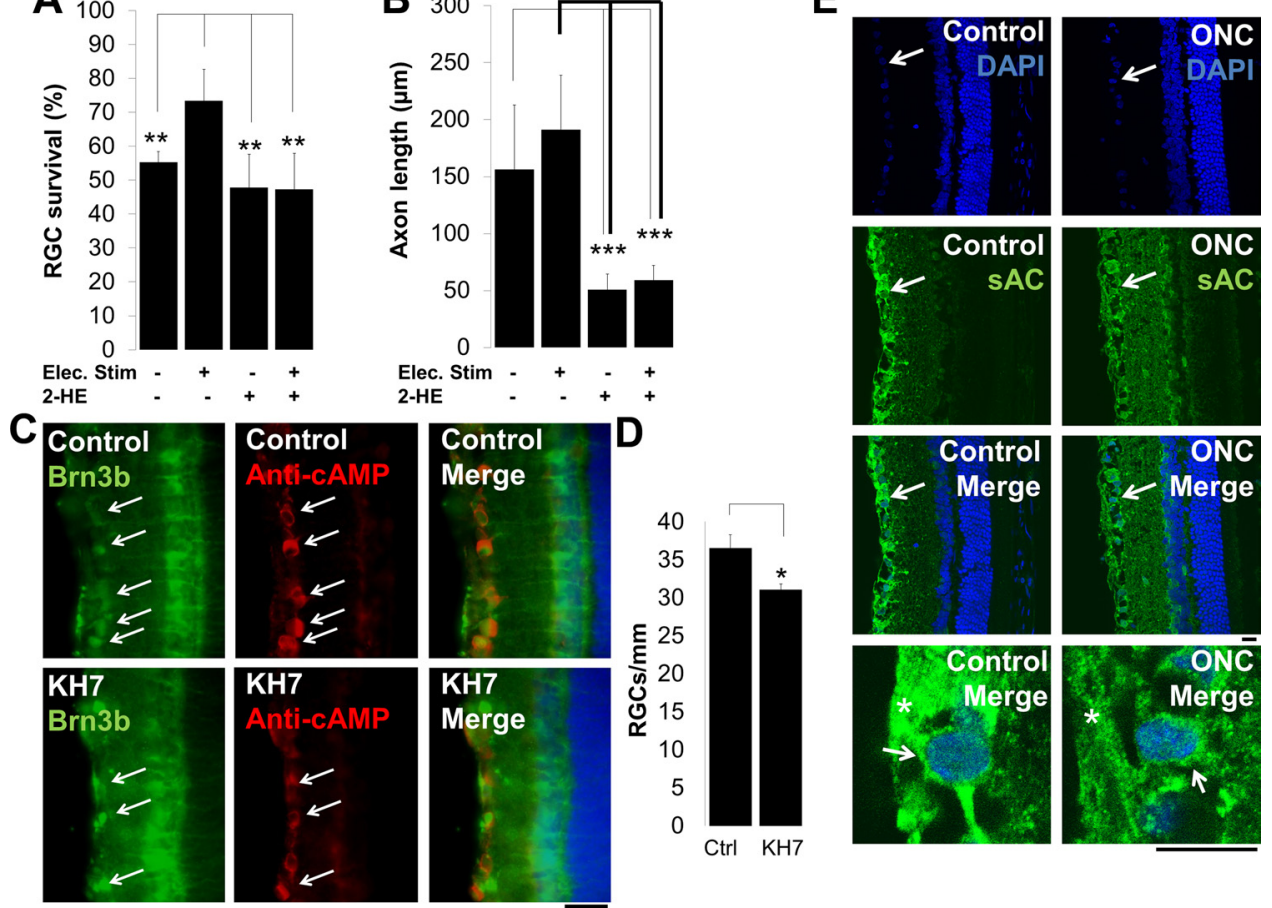

Figure 8. $s \mathrm{AC}$ activity is required for $\mathrm{RGC}$ survival and axon growth in response to electrical stimulation in vitro and survival in vivo. $A, B, \operatorname{In}$ the presence of maximal $s \mathrm{AC}$ and tm $\mathrm{AC}$ stimulation with bicarbonate and forskolin, electrical stimulation significantly increases RGC survival $(\boldsymbol{A})$ but not axon growth $(\boldsymbol{B})$. Inhibiting $\mathrm{SAC}$ with 2 -HE abrogates the survival effect induced by electrical stimulation (A), and RGC axon growth independent of electrical stimulation (B). Error bars, SD; $N=5$, ANOVA, ${ }^{*} p<0.05,{ }^{* *} p<0.01,{ }^{* * *} p<0.001$. C, Immunostaining retinal sections for RGCS

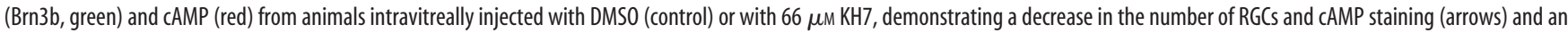
atrophic appearance. Scale bar, $25 \mu \mathrm{m}$. D, Quantification shows a significant decrease in RGCs after KH7 injection (error bars, SD; $n=4$ animals per group, $t$ test, ${ }^{*} p<0.05$ ). $\boldsymbol{E}$, sAC immunoreactivity decreases after optic nerve injury. Control retinas (left) were compared with retinas obtained $6 \mathrm{~d}$ after optic nerve crush (right). $\mathrm{SAC}$ staining (green) in control retinas is more intense in the ganglion cell layer (arrows) and nerve fiber layer (asterisk) compared with injured retinas. Scale bars, $50 \mu \mathrm{m}$.

et al., 1998; Zhang and Poo, 2001; Goldberg et al., 2002). Our data on sAC activation suggests at least an additive activity with tmACs, which is corroborated by the poor effect of forskolin in promoting RGC survival and growth in the absence of bicarbonate (Fig. 5).
Similarly, neither pharmacological tmAC inhibition nor AC1/AC8 $\mathrm{KO}$ affected RGC survival or growth in vitro or in vivo (Nicol et al., 2006a, 2007) raising a hypothesis that for RGC survival and growth $\mathrm{sAC}$ may be prove to be more important than tmACs. 
Although the possibility of other tmAC compensating for $\mathrm{ACl} / \mathrm{AC} 8$ in the $\mathrm{KO}$ animal is certainly possible, RGCs have only three ACs activated by calcium (AC1, AC8 and $\mathrm{sAC}$ ) and 6 total tmACs (AC1, AC2, AC3, AC5, AC8, and AC9) (Nicol et al., 2006b). The role of AC1 and AC8 has been extensively characterized using knock-out animals and shows that $\mathrm{AC} 1$ and $\mathrm{AC} 8$ are important for axon guidance and growth cone collapse (Nicol et al., 2006a, 2007, 2011). AC1/AC8 animals do not show an increase in baseline RGC survival, although those animals are more susceptible to neuronal death after insults like ethanol during the neonatal period (Conti et al., 2009), consistent with our own in vitro data from the double knock-out animals in Figure 7. Thus our and others' data demonstrate that AC1/AC8 are not necessary for RGC survival under basal conditions.

\section{cAMP specificity may require compartmentalized signaling}

How is a single molecule important for such diverse, yet specific cellular processes? Spatial and temporal compartmentalization are the most important mechanisms described (Grandoch et al., 2010). Compartmentalization is created enzymatically by subcellular localization of cAMP-producing ACs and cAMP-degrading phosphodiesterases, as well as by anchoring proteins such as A-kinase anchoring proteins, organized in protein complexes restricted to specific subcellular subdomains (Dessauer, 2009; Christian et al., 2011). This specification restricts cAMP diffusion to subcellular domains such as the growth cone (Shelly et al., 2010), mitochondria, the endoplasmic reticulum and nuclear membranes (Zippin et al., 2004; Acin-Perez et al., 2009a), likely enhancing compartmentalization. Compartmentalization, in turn, limits signaling cascade activation/propagation generating molecular oscillations (Corredor and Goldberg, 2009; Dunn et al., 2009). sAC localizes to multiple intracellular compartments including the nucleus, cytoplasm and mitochondria in several cell types (Feng et al., 2005; Ramos et al., 2008; Acin-Perez et al., 2009a; Geng et al., 2009; Hallows et al., 2009; Strazzabosco et al., 2009; Zippin et al., 2010; Li et al., 2011). Our data show that sAC protein localizes to the nucleus, cytoplasm, and central domain of the growth cone supporting a compartmentalized signaling model. However, sAC function, isoform types, and interaction between isoforms in each intracellular compartment remain to be elucidated.

sAC's localization to multiple compartments in RGCs, including the nucleus, central cytoplasm, and peripheral neurites, is compatible with suggested sAC functions in transcription (Zippin et al., 2010) and mitochondrial regulation (Acin-Perez et al., 2009a,b). Mitochondria are impermeable to cytoplasmic cAMP, so $\mathrm{SAC}$ is the only source of intramitochondrial cAMP (AcinPerez et al., 2009a). It is possible that survival can be partially compensated for by other sources of cytosolic cAMP derived from tmACs at the lower doses of $\mathrm{KH} 7$, while at higher doses the complete inhibition of mitochondrial or other sAC induces cell death. This may similarly reflect a compartmentalized effect of different sAC roles in cellular physiology.

Since we detected only one sAC isoform by Western blot with an antibody raised against the catalytic domain, the question arises whether multiple cellular functions may be achieved by a single catalytically active isoform. This would be possible in a scenario where compartment-specific protein-protein interactions determine specific functions for just one isoform; however, we cannot rule out the presence of lower levels of other functional isoforms. In fact, transcriptome analysis of rat RGCs indicated that multiple Sac isoforms, some of them expressed at very low levels, are present in RGCs (Fig. 4), including some isoforms with only the C terminus. Interestingly, we identified, cloned and sequenced another novel Sac splicing isoform missing $192 \mathrm{bp}$ from the presumed ATPase domain (Fig. 4), but did not further characterize the $\mathrm{N}$ and $\mathrm{C}$ termini. Intriguingly, the somatic sAC isoform is proposed to have only one catalytic domain; tmACs and the testis sAC isoforms have two (Xie and Conti, 2004). If two catalytic domains are required for CAMP synthesis, the single SAC catalytic domain may form functional homodimers or heterodimers with other AC catalytic domains, as has been described for tmACs (Baragli et al., 2008). Nevertheless, since sAC pharmacologic inhibitors target the catalytic domain(s) and have high membrane permeability, it is expected that all cAMPsynthesizing isoforms should be blocked by these compounds, whatever their subcellular localization. Overexpressing subcellularly directed SAC isoforms in a sAC knockdown background may help address whether sAC activity in specific compartments mediates sAC's effect on RGC survival, axon growth, or calcium/ cAMP oscillations, although constitutively active sAC mutants have not yet been described.

\section{sAC activation is necessary and sufficient to increase} intracellular cAMP, survival and axon growth in RGCs

Our observation that electrical activity promotes RGC survival even in the presence of forskolin, a tmAC activator, including all calcium-sensitive tmACs (Strazzabosco et al., 2009), led us to the hypothesis that calcium-sensitive sAC is an additional source of cAMP in RGCs. The additional effect of electrical stimulation over forskolin in promoting RGC survival and axon growth in response to BDNF is blocked by SAC inhibition. Electrical stimulation-induced cAMP elevation in RGCs has been demonstrated by us and others (Dunn et al., 2009); considering variables like stimulus frequency, intensity and duration may provide new insight into the use of electrical stimulation for scientific and therapeutic use (Corredor and Goldberg, 2009).

Given sAC's role in RGC survival and axon growth, understanding the mechanisms for sAC's physiologic activation remains an important area for study. We and others demonstrated that stimulating electrical activity at physiologic levels strongly promotes RGC survival and axon growth in vitro and in vivo, and that this effect depends on calcium entry, cAMP elevation, and PKA activation (Meyer-Franke et al., 1998; Shen et al., 1999; Goldberg et al., 2002; Morimoto et al., 2005; Corredor and Goldberg, 2009; Tagami et al., 2009), and here we show dependence on sAC activity. We also demonstrated that the sAC activator bicarbonate increased intracellular cAMP and promoted axon growth and survival in RGCs. sAC activators bicarbonate and calcium synergize to activate sAC by increasing sAC's $V_{\max }$ and substrate affinity, respectively (Litvin et al., 2003). Like calcium, bicarbonate participates in many physiological processes (Casey et al., 2009). Retinal cells express bicarbonate transporters and bicarbonate-synthesizing enzymes like carbonic anhydrases (Adamus and Karren, 2009; Casey et al., 2009). Krebs cyclederived bicarbonate activates mitochondrial sAC, which is a critical metabolic sensor and modulates oxidative phosphorylation (Acin-Perez et al., 2009a,b). The potent $\mathrm{pH}$ buffering capacity of bicarbonate limits its therapeutic application in vivo, but these data encourage the search for alternative approaches to activate sAC (including electrical stimulation), and motivate further investigating this complex cell signaling cascade with promising therapeutic implications. 


\section{References}

Acin-Perez R, Salazar E, Kamenetsky M, Buck J, Levin LR, Manfredi G (2009a) Cyclic AMP produced inside mitochondria regulates oxidative phosphorylation. Cell Metab 9:265-276.

Acin-Perez R, Salazar E, Brosel S, Yang H, Schon EA, Manfredi G (2009b) Modulation of mitochondrial protein phosphorylation by soluble adenylyl cyclase ameliorates cytochrome oxidase defects. EMBO Mol Med 1:392-406.

Adamus G, Karren L (2009) Autoimmunity against carbonic anhydrase II affects retinal cell functions in autoimmune retinopathy. J Autoimmun 32:133-139.

Baragli A, Grieco ML, Trieu P, Villeneuve LR, Hébert TE (2008) Heterodimers of adenylyl cyclases 2 and 5 show enhanced functional responses in the presence of Galpha s. Cell Signal 20:480-492.

Casey JR, Sly WS, Shah GN, Alvarez BV (2009) Bicarbonate homeostasis in excitable tissues: role of $\mathrm{AE} 3 \mathrm{Cl}-/ \mathrm{HCO} 3$-exchanger and carbonic anhydrase XIV interaction. Am J Physiol Cell Physiol 297:C1091-C1102.

Chen MH, Chen H, Zhou Z, Ruan YC, Wong HY, Lu YC, Guo JH, Chung YW, Huang PB, Huang HF, Zhou WL, Chan HC (2010) Involvement of CFTR in oviductal HCO3-secretion and its effect on soluble adenylate cyclase-dependent early embryo development. Hum Reprod 25:1744-1754.

Christian F, Szaszák M, Friedl S, Drewianka S, Lorenz D, Goncalves A, Furkert J, Vargas C, Schmieder P, Götz F, Zühlke K, Moutty M, Göttert H, Joshi M, Reif B, Haase H, Morano I, Grossmann S, Klukovits A, Verli J, et al. (2011) Small molecule AKAP-protein kinase A (PKA) interaction disruptors that activate PKA interfere with compartmentalized cAMP signaling in cardiac myocytes. J Biol Chem 286:9079-9096.

Conti AC, Maas JW Jr, Moulder KL, Jiang X, Dave BA, Mennerick S, Muglia LJ (2009) Adenylyl cyclases 1 and 8 initiate a presynaptic homeostatic response to ethanol treatment. PLoS One 4:e5697.

Corredor RG, Goldberg JL (2009) Electrical activity enhances neuronal survival and regeneration. J Neural Eng 6:55001.

Dapson R (2007) Effects of fixation on routine, special and immunohistochemical stains: introduction to a symposium for the Biological Stain Commission. Biotech Histochem Oct 3:1-2.

Dessauer CW (2009) Adenylyl cyclase-A-kinase anchoring protein complexes: the next dimension in cAMP signaling. Mol Pharmacol 76:935-941.

Dunn TA, Storm DR, Feller MB (2009) Calcium-dependent increases in protein kinase-A activity in mouse retinal ganglion cells are mediated by multiple adenylate cyclases. PLoS One 4:e7877.

Farrell J, Ramos L, Tresguerres M, Kamenetsky M, Levin LR, Buck J (2008) Somatic 'soluble' adenylyl cyclase isoforms are unaffected in Sacy tm1Lex/Sacy tm1Lex 'knockout' mice. PLoS One 3:e3251.

Feng QP, Zuo J, Meng Y, Fang FD (2005) [Nuclear localization region in soluble adenylyl cyclase]. Zhongguo Yi Xue Ke Xue Yuan Xue Bao 27:280-284

Geng W, Wang Z, Zhang J, Reed BY, Pak CY, Moe OW (2005) Cloning and characterization of the human soluble adenylyl cyclase. Am J Physiol Cell Physiol 288:C1305-C1316.

Geng W, Hill K, Zerwekh JE, Kohler T, Müller R, Moe OW (2009) Inhibition of osteoclast formation and function by bicarbonate: role of soluble adenylyl cyclase. J Cell Physiol 220:332-340.

Goldberg JL, Espinosa JS, Xu Y, Davidson N, Kovacs GT, Barres BA (2002) Retinal ganglion cells do not extend axons by default: promotion by neurotrophic signaling and electrical activity. Neuron 33:689-702.

Grandoch M, Roscioni SS, Schmidt M (2010) The role of Epac proteins, novel cAMP mediators, in the regulation of immune, lung and neuronal function. Br J Pharmacol 159:265-284.

Hallows KR, Wang H, Edinger RS, Butterworth MB, Oyster NM, Li H, Buck J, Levin LR, Johnson JP, Pastor-Soler NM (2009) Regulation of epithelial $\mathrm{Na}+$ transport by soluble adenylyl cyclase in kidney collecting duct cells. J Biol Chem 284:5774-5783.

Han H, Stessin A, Roberts J, Hess K, Gautam N, Kamenetsky M, Lou O, Hyde E, Nathan N, Muller WA, Buck J, Levin LR, Nathan C (2005) Calciumsensing soluble adenylyl cyclase mediates TNF signal transduction in human neutrophils. J Exp Med 202:353-361.

Hess KC, Jones BH, Marquez B, Chen Y, Ord TS, Kamenetsky M, Miyamoto C, Zippin JH, Kopf GS, Suarez SS, Levin LR, Williams CJ, Buck J, Moss SB (2005) The "soluble" adenylyl cyclase in sperm mediates multiple signaling events required for fertilization. Dev Cell 9:249-259.
Jaiswal BS, Conti M (2001) Identification and functional analysis of splice variants of the germ cell soluble adenylyl cyclase. J Biol Chem 276:31698-31708.

Kumar S, Kostin S, Flacke JP, Reusch HP, Ladilov Y (2009) Soluble adenylyl cyclase controls mitochondria-dependent apoptosis in coronary endothelial cells. J Biol Chem 284:14760-14768.

Li S, Allen KT, Bonanno JA (2011) Soluble adenylyl cyclase mediates bicarbonate-dependent corneal endothelial cell protection. Am J Physiol Cell Physiol 300:C368-C374.

Litvin TN, Kamenetsky M, Zarifyan A, Buck J, Levin LR (2003) Kinetic properties of "soluble" adenylyl cyclase. Synergism between calcium and bicarbonate. J Biol Chem 278:15922-15926.

Lomovatskaya LA, Romanenko AS, Filinova NV, Salyaev RK (2008) Detection of soluble adenylyl cyclase isoforms in plants. Dokl Biochem Biophys 420:124-126.

Meyer-Franke A, Kaplan MR, Pfrieger FW, Barres BA (1995) Characterization of the signaling interactions that promote the survival and growth of developing retinal ganglion cells in culture. Neuron 15:805-819.

Meyer-Franke A, Wilkinson GA, Kruttgen A, Hu M, Munro E, Hanson MG Jr, Reichardt LF, Barres BA (1998) Depolarization and cAMP elevation rapidly recruit TrkB to the plasma membrane of CNS neurons. Neuron 21:681-693.

Miotke JA, MacLennan AJ, Meyer RL (2007) Immunohistochemical localization of CNTFRalpha in adult mouse retina and optic nerve following intraorbital nerve crush: evidence for the axonal loss of a trophic factor receptor after injury. J Comp Neurol 500:384-400.

Morimoto T, Miyoshi T, Matsuda S, Tano Y, Fujikado T, Fukuda Y (2005) Transcorneal electrical stimulation rescues axotomized retinal ganglion cells by activating endogenous retinal IGF-1 system. Invest Ophthalmol Vis Sci 46:2147-2155.

Murray AJ, Shewan DA (2008) Epac mediates cyclic AMP-dependent axon growth, guidance and regeneration. Mol Cell Neurosci 38:578-588.

Nicol X, Muzerelle A, Rio JP, Métin C, Gaspar P (2006a) Requirement of adenylate cyclase 1 for the ephrin-A5-dependent retraction of exuberant retinal axons. J Neurosci 26:862-872.

Nicol X, Bennis M, Ishikawa Y, Chan GC, Repérant J, Storm DR, Gaspar P (2006b) Role of the calcium modulated cyclases in the development of the retinal projections. Eur J Neurosci 24:3401-3414.

Nicol X, Voyatzis S, Muzerelle A, Narboux-Nême N, Südhof TC, Miles R, Gaspar P (2007) cAMP oscillations and retinal activity are permissive for ephrin signaling during the establishment of the retinotopic map. Nat Neurosci 10:340-347.

Nicol X, Hong KP, Spitzer NC (2011) Spatial and temporal second messenger codes for growth cone turning. Proc Natl Acad Sci USA 108:13776-13781.

Pastor-Soler N, Beaulieu V, Litvin TN, Da Silva N, Chen Y, Brown D, Buck J, Levin LR, Breton S (2003) Bicarbonate-regulated adenylyl cyclase (sAC) is a sensor that regulates $\mathrm{pH}$-dependent $\mathrm{V}$-ATPase recycling. J Biol Chem 278:49523-49529.

Păunescu TG, Ljubojevic M, Russo LM, Winter C, McLaughlin MM, Wagner CA, Breton S, Brown D (2010) cAMP stimulates apical V-ATPase accumulation, microvillar elongation, and proton extrusion in kidney collecting duct A-intercalated cells. Am J Physiol Renal Physiol 298:F643-F654.

Ramos LS, Zippin JH, Kamenetsky M, Buck J, Levin LR (2008) Glucose and GLP-1 stimulate cAMP production via distinct adenylyl cyclases in INS-1E insulinoma cells. J Gen Physiol 132:329-338.

Root CM, Velázquez-Ulloa NA, Monsalve GC, Minakova E, Spitzer NC (2008) Embryonically expressed GABA and glutamate drive electrical activity regulating neurotransmitter specification. J Neurosci 28:4777-4784.

Schlicker C, Rauch A, Hess KC, Kachholz B, Levin LR, Buck J, Steegborn C (2008) Structure-based development of novel adenylyl cyclase inhibitors. J Med Chem 51:4456-4464.

Schmid A, Sutto Z, Nlend MC, Horvath G, Schmid N, Buck J, Levin LR, Conner GE, Fregien N, Salathe M (2007) Soluble adenylyl cyclase is localized to cilia and contributes to ciliary beat frequency regulation via production of cAMP. J Gen Physiol 130:99-109.

Shelly M, Lim BK, Cancedda L, Heilshorn SC, Gao H, Poo MM (2010) Local and long-range reciprocal regulation of cAMP and cGMP in axon/dendrite formation. Science 327:547-552.

Shen S, Wiemelt AP, McMorris FA, Barres BA (1999) Retinal ganglion cells lose trophic responsiveness after axotomy. Neuron 23:285-295. 
Song HJ, Ming GL, Poo MM (1997) cAMP-induced switching in turning direction of nerve growth cones. Nature 388:275-279.

Spitzer NC (2006) Electrical activity in early neuronal development. Nature 444:707-712.

Steegborn C, Litvin TN, Levin LR, Buck J, Wu H (2005a) Bicarbonate activation of adenylyl cyclase via promotion of catalytic active site closure and metal recruitment. Nat Struct Mol Biol 12:32-37.

Steegborn C, Litvin TN, Hess KC, Capper AB, Taussig R, Buck J, Levin LR, Wu H (2005b) A novel mechanism for adenylyl cyclase inhibition from the crystal structure of its complex with catechol estrogen. J Biol Chem 280:31754-31759.

Stessin AM, Zippin JH, Kamenetsky M, Hess KC, Buck J, Levin LR (2006) Soluble adenylyl cyclase mediates nerve growth factor-induced activation of Rap1. J Biol Chem 281:17253-17258.

Strazzabosco M, Fiorotto R, Melero S, Glaser S, Francis H, Spirli C, Alpini G (2009) Differentially expressed adenylyl cyclase isoforms mediate secretory functions in cholangiocyte subpopulation. Hepatology 50:244-252.

Tagami Y, Kurimoto T, Miyoshi T, Morimoto T, Sawai H, Mimura O (2009) Axonal regeneration induced by repetitive electrical stimulation of crushed optic nerve in adult rats. Jpn J Ophthalmol 53:257-266.

Topal H, Fulcher NB, Bitterman J, Salazar E, Buck J, Levin LR, Cann MJ, Wolfgang MC, Steegborn C (2012) Crystal structure and regulation mechanisms of the CyaB adenylyl cyclase from the human pathogen Pseudomonas aeruginosa. J Mol Biol 416:271-286.

Tresguerres M, Parks SK, Salazar E, Levin LR, Goss GG, Buck J (2010) Bicarbonate-sensing soluble adenylyl cyclase is an essential sensor for acid/base homeostasis. Proc Natl Acad Sci U S A 107:442-447.

Usher LC, Johnstone A, Ertürk A, Hu Y, Strikis D, Wanner IB, Moorman S, Lee JW, Min J, Ha HH, Duan Y, Hoffman S, Goldberg JL, Bradke F, Chang YT, Lemmon VP, Bixby JL (2010) A chemical screen identifies novel compounds that overcome glial-mediated inhibition of neuronal regeneration. J Neurosci 30:4693-4706.

Wang Y, Lam CS, Wu F, Wang W, Duan Y, Huang P (2005) Regulation of CFTR channels by $\mathrm{HCO}(3)$-sensitive soluble adenylyl cyclase in human airway epithelial cells. Am J Physiol Cell Physiol 289:C1145-C1151.

Wong ST, Athos J, Figueroa XA, Pineda VV, Schaefer ML, Chavkin CC, Muglia LJ, Storm DR (1999) Calcium-stimulated adenylyl cyclase activity is critical for hippocampus-dependent long-term memory and late phase LTP. Neuron 23:787-798.

Wu KY, Zippin JH, Huron DR, Kamenetsky M, Hengst U, Buck J, Levin LR, Jaffrey SR (2006) Soluble adenylyl cyclase is required for netrin-1 signaling in nerve growth cones. Nat Neurosci 9:1257-1264.

Xie F, Conti M (2004) Expression of the soluble adenylyl cyclase during rat spermatogenesis: evidence for cytoplasmic sites of cAMP production in germ cells. Dev Biol 265:196-206.

Yamada RX, Matsuki N, Ikegaya Y (2005) cAMP differentially regulates axonal and dendritic development of dentate granule cells. J Biol Chem 280:38020-38028.

Zhang LI, Poo MM (2001) Electrical activity and development of neural circuits. Nat Neurosci 4 [Suppl]:1207-1214.

Zhao L, Sheng AL, Huang SH, Yin YX, Chen B, Li XZ, Zhang Y, Chen ZY (2009) Mechanism underlying activity-dependent insertion of TrkB into the neuronal surface. J Cell Sci 122:3123-3136.

Zippin JH, Farrell J, Huron D, Kamenetsky M, Hess KC, Fischman DA, Levin LR, Buck J (2004) Bicarbonate-responsive "soluble" adenylyl cyclase defines a nuclear cAMP microdomain. J Cell Biol 164:527-534.

Zippin JH, Chadwick PA, Levin LR, Buck J, Magro CM (2010) Soluble adenylyl cyclase defines a nuclear cAMP microdomain in keratinocyte hyperproliferative skin diseases. J Invest Dermatol 130:1279-1287. 